\title{
Research on Intelligent CNC Turret Punch Press Process Programming
}

\section{System}

\author{
Cao Ai-xia ${ }^{1, a^{*}}$, Chen Jiang-bo ${ }^{1}$ \\ ${ }^{1}$ Qingdao Huanghai University, College of Traffic and Ship Engineering, Shandong Qingdao \\ 266427, China \\ acaoaixia1123@163.com
}

\begin{abstract}
With the continuous development of industrial technology in China, current producting mechanical equipment and tools with high quality, is the most important project in manufacturing industry, so as to effectively serve the other production industry, effectively use modern technology to improve the level of social production, and improve the efficiency of the national economy. The core of the equipment manufacturing industry is CNC punching equipment, one of the main types of CNC turret punch press is the CNC punch. In the study, the formation and characteristics of CNC turret punch were analyzed, and the intelligent process programming system were researched, to make CNC turret punch press more intelligently, and promote the development of China's manufacturing industry.
\end{abstract}

Key words: CNC turret punch press, Intelligent, Process programming system, Research.

\section{Introduction}

Before the birth of CNC turret punch press, stamping processing work used to be finished by pressure. In the work, the use of pressure was more suitable for processing a large number of artifacts, which was in the same style. And for producting small batches and various varieties of workpiece, the workers spend a lot of time to change mould, and this would reduce the machining efficiency, and mould cost also would increase. With the continuous development of science and technology, in 1934, American Wiedemann inc. designed the world's first R2 turret punch press, also called manual multi-station punch press, which is the predecessor of CNC turret punch press. In 1964 Wiedemann inc. was acquired by Warner \& Swasey inc. Until on the international machine tool show in Chicago, America in 1970, Warner \& Swasey inc. showed the first CNC turret punch press in the world.

The intelligent CNC turret punch press process programming principle of practical work

CNC turret punch press depends on the advantage of flexible programming, free choice model, rapid, is now popular in the users. And with the continuous development of science and technology in our country, it also provides upgrading of CNC turret punch press with very favorable conditions. CNC turret punch press is a kind of stamping equipment technology with high efficiency, high precision and general. It has more than one location, and it can realize quick die change in work. CNC turret punch press is mainly composed by numerical control system, electrical system, servo system, lathe bed and the basic auxiliary device and the traditional system. During the machining using CNC turret punch press, mainly on sheet forming, the construction of the plate thickness is about $0.5 \sim 6 \mathrm{~mm}$, effectively realize the function of implementation on plate punching, stretching, and forming etc. Function. Before the construction of CNC turret punch press, turret is the mould 
machine tool library, which contains different mould style, while in the stamping die with lower die, so the turret also is divided into upper and lower turret, turn on mould installation in tower, under the will of the mould installation on a turret, and bolted, so as to ensure the effective implementation of the CNC turret punch press work. For X, Y axis servo system and transmission mechanism of turret punch press, can finish all the work project under the numerical control technology. CNC turret punch press in the work, machine tool clamp system will plate fixed on the workbench, in decoding the nc machining program by numerical control system, the output control signal, real-time remote control effectively for each axis, guarantee the correct operation, using the fixed clamp plate can pinpoint when processing, security system and implemented according to the control system the directives issued by the relevant job content, and then complete the punching, and the whole process is accomplished in accordance with the order of the nc code layout. CNC turret punch press mainly in the two-dimensional plane point for processing, and efficient in work CNC turret punch press speed can reach $100 \sim 150 \mathrm{r} / \mathrm{min}$, so when making CNC turret punch press machining, must guarantee sheet stamping process accurate fixed position, so as to guarantee the real time and efficiency of numerical control system, so as to complete the higher request, to promote the sound development in the manufacturing industry.

\section{The design of hardware and software system in CNC turret punch press}

(1) The design of CNC turret punch press hardware system

The CNC turret punch press control system hardware, mainly composed by the development-oriented programmable PC remote control card, it is a common method in the numerical control system. And the use of remote control card mainly in the processing machine tool remote remote control, input and output signal detection, such as work, and the real-time requirement is relatively high. For PC, the main work is to give related to motion control by remote data, effective access to current motion state, and in the use of interactive technology to display, it is main or daemon, the real-time requirements are not particularly high. Figure 1 shows the effective of open CNC system structure, it consists of industrial computer remote control card - remote actuators - servo drive and servo motor - machine system of organizations such as the input and output.

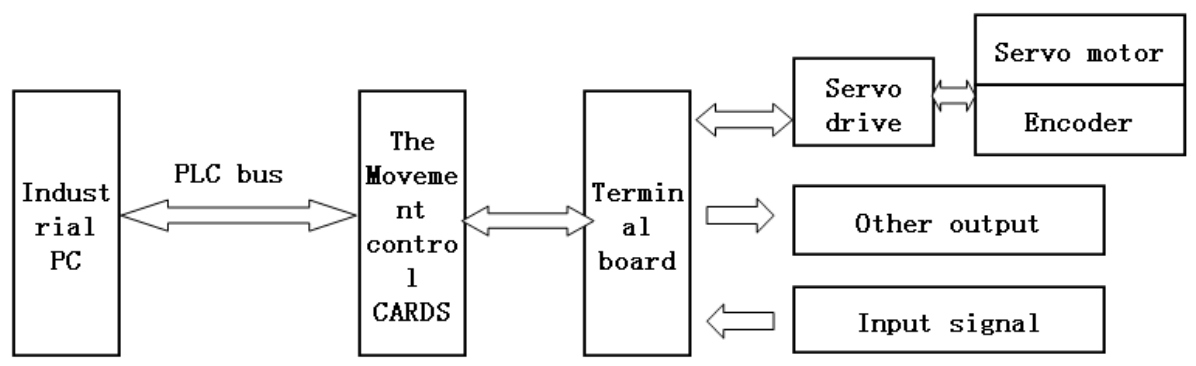

Figure.1 open CNC system structure

Based on CNC turret punch press demand for servo control system is analyzed, figure 2 is a control system, the hardware structure of the remote control of the PC and remote control function, and the remote controller is the core part of its control, can effectively the interpolation operation of the motor, position control, signal detection and other remote I/O functions, then the PC part of data processing and human-computer interaction function effectively, in the use of PCI bus communication link between the two, in accordance with the relevant requirements after connecting 
plate and the digital quantity expansion mould fast in remote extension card access to the signal transfer, for connected to the later work well.

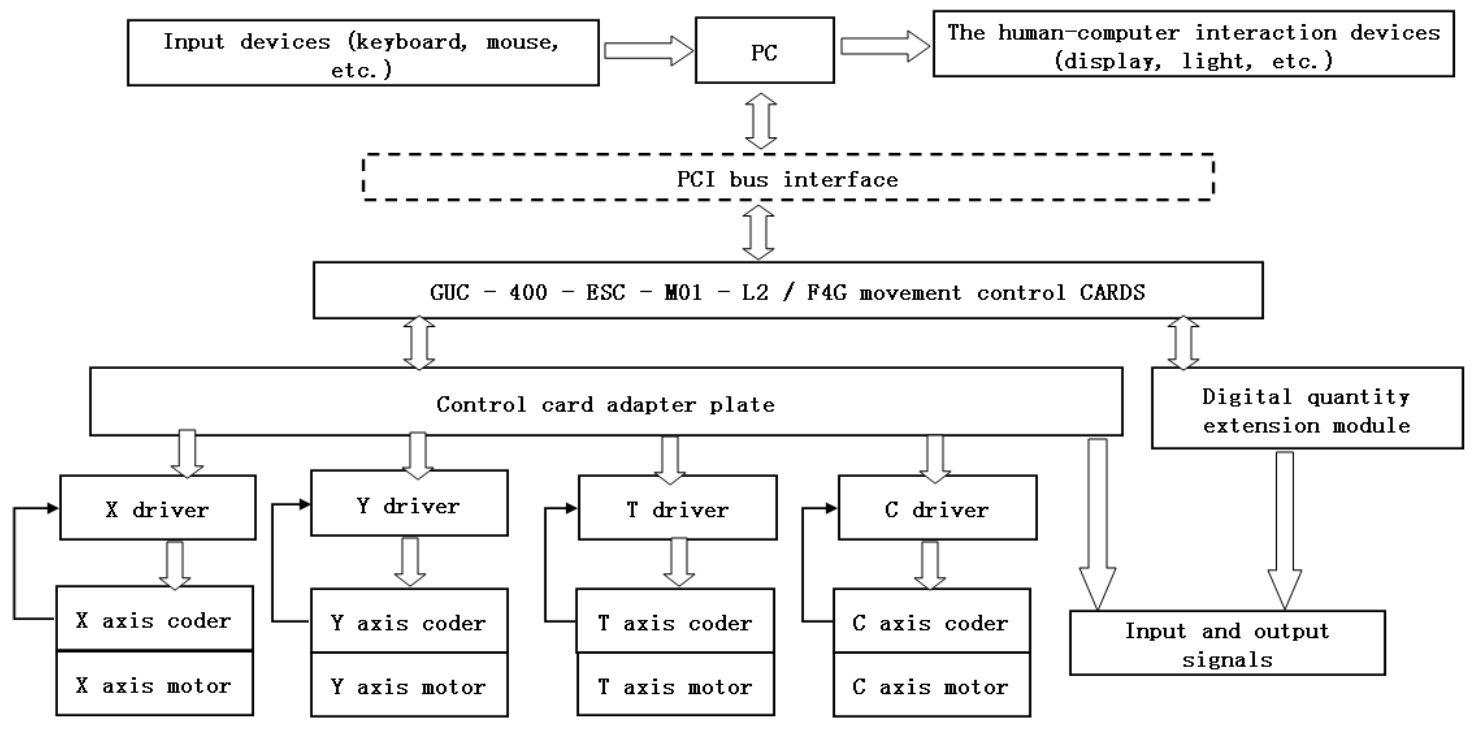

Figure.2 control system hardware structure diagram

(2) The design of CNC turret punch press software system

In the hardware system of CNC turret punch press, CNC equipment is the basis of machining parts, and it is the executor of the implementation of remote and actions. For CNC turret punch press, software is the conductor in the process of machining, but also is embodied in the numerical control equipment performance is a key part of the high and low. For hardware system, the tower load numerical control system is different, the processing ability also will be different, so the numerical control equipment are in need of a stable, functional equipment reasonable system, so as to develop the function of each part, so as to improve the work efficiency of CNC turret punch press.

\section{The main functions of the CNC turret punch press and its technological design}

(1) stroke control function

Stroke control is mainly used on the machining of workpieces, in use of NC machine tool fixed position of the output signal to activate the punch, and impact load cell is through the operation of the input signal response on the NC punch, and in this time is not allowed any axis running, can only be carried out until after the impact load is positioning work again.

(2) the fast signal function

In CNC turret punch press work, in order to be able to realize digital control and synchronization between the impact load unit, effective son are used in the function of fast signal, their role first in only when plate stops, can through the quick release stroke output. Second is when the blunt knife and sheet metal contact, using fast input to keep the sheet in stop state, the normal CNC turret punch press can thus guaranteed.

(3) the release function of stroke

In CNC turret punch press work, stroke is released at the earliest to ensure feed axis static Settings, it can effectively guarantee the impact load, the relative movement between the punch and machining of plane plate does not happen, to ensure normal operation in CNC turret punch press.

The upgrading of intelligent $\mathrm{CNC}$ turret punch press process programming

(1) the first generation of CNC turret punch press machinery 
The CNC machinery is mainly the turret punch press machine, the mechanical transmission is composed by the flywheel, the crank and slide block mechanism, a three-phase asynchronous motor, flywheel, clutch (brake), crankshaft, connecting rod, sliding block equipment, etc. For this kind of machine tool, its advantage is simple structure, convenient maintenance in the future, but low price of the product is also more stable performance, service life is long. But it also has certain drawbacks, numerical control machine turret punch press in the completion of a stamping must wait for flywheel turned to after a circle, because the stamping stroke is fixed, the stamping frequency is too low, can't effectively enhance general stamping speed. And because the blow head lack certain controlling schedule and that makes for stamping molding process, cannot easily be controlled, and this will lead to forming effect is not ideal, the processing technology will be affected by different factors, and power consumption of this kind of machine is bigger, also has the serious noise problems.

(2) of the second generation of CNC hydraulic turret punch press

Along with the advance of science and technology, the second generation of CNC turret punch press also gradually entered people's field of vision, it is the main hydraulic power on CNC turret punch press, hydraulic main transmission by using hydraulic power transmission instead of the first generation of numerical control machine turret punch press, hydraulic system of main transmission is by the control unit, hydraulic power pack, hydraulic station and hydraulic line part of the organization, the job is hydraulic pressure station and hydraulic power head is connect hydraulic line, after the three-phase asynchronous motor to drive the gear pump in the hydraulic station to provide hydraulic power head hydraulic oil which by the control unit to control different valve in hydraulic power head, effectively under the hydraulic power head movement and related work, and finally realize the punch position, speed, driving, etc for accurate and precise control of the work. CNC hydraulic turret punch press, the advantages of it by hydraulic cylinder to drive the blow head, in order to realize the stamping movement, working content, after by hydraulic power head and position sensor to cooperate, so as to control the stamping work. CNC hydraulic turret punch press can effectively extend the CNC turret punch press overall processing technology, can effectively remove overload and bring damage to machine tools.

(3) the third generation of NC servo turret punch press

For CNC turret punch press, as a result of the numerical control mechanical and hydraulic turret punch press can't satisfy the user's machine high speed, low energy consumption, low noise, such as requirements, now the application of servo motor technology and development, to the third generation of CNC turret punch press with good development condition, the numerical control servo turret punch press is gradually accepted by people. And the advantages of nc servo turret punch press is also more, for one, has the function of can achieve energy-saving noise reduction of nc servo turret punch press is driven by servo motor for main transmission, unlike mechanical, it does not have the flywheel, so there is no need to accumulate energy, only in the stamping in the servo motor is rotating, and at the completion of a stamping, before sent to the opening sheet, servo motor has been remain static. And because of remove the clutch and brake, effective shorten transmission mechanism, the CNC turret punch press at work to reduce the consumption of electricity has a very big help. And compared with the CNC hydraulic turret punch press, CNC servo turret punch press power consumption is also only a third of the CNC hydraulic turret punch press. Second, can effectively improve the working efficiency of machine tool, in the midst of CNC turret punch press with slider velocity can be arbitrary adjustment and control in the whole journey, in the work be able to choose the best by punching process of different punch stroke, and formulate the corresponding punching speed according to corresponding requirements, to ensure the 
consistent with the conveying mechanism can, thus enhances the working efficiency of the machine tool. Third, can effectively optimize the shallow stretch forming process, and improve the process precision and stability. In numerical control servo turret punch press can accurately set the position of the slider, can effectively improve molding processing precision, through setting height of molding, to guarantee the stability in the process of forming.

\section{Conclusion}

With the continuous development of industrialization technology in our country, The CNC turret punch press technology in China are also in the continuous development and progress. The CNC turret punch press through making the continuous research, improvement, effectively using of intelligent process programming system to provide a very favorable condition for China's manufacturing industry development. It effectively promotes the economic development of manufacturing industry in our country, and the stability of the national economy progress.

\section{Acknowledgements}

This research was financially supported by the Science and technology project of Shandong Province (J15LB55) and the project of domestic visiting scholars of young backbone teachers of Shandong Province.

\section{References}

[1] Wen-long wang; Pei-si zhong. Han Yingying; Li Guili; Liu mei. Turret punch press machining process planning and nc programming research [J]. Journal of mechanical design and manufacturing, 2013 (5) : 237-238.

[2] Wang. Turret punching machine nc system and other key technologies of design and research [D]. Xiamen university, 2007 (5) : master's thesis.

[3] Zhu Yu; Wei Zhiguo. Shallow of the development of CNC turret punch press [J]. Journal of forging equipment and manufacturing technology, 2016 (2) : 59-61.

[4] Yang cp; SiHongBo. CNC turret punch press technical transformation [J]. Journal of the science and technology, 2013 (7) : 142-143.

[5] John Bruce yeh. Lin turret punch press the design of the CNC system [D]. Xiamen university, 2009 (5) : master's thesis

[6] Wen-long wang. Key techniques of CNC turret punch press process programming system [D]. Shandong university of science and technology, 2012 (5) : master's thesis. 